\title{
Phantom and clinical evaluation of bone SPECT/CT image reconstruction with xSPECT algorithm
}

\author{
Noriaki Miyaji ${ }^{1,2}$, Kenta Miwa $^{3}$, Ayaka Tokiwa ${ }^{3}$, Hajime Ichikawa ${ }^{4}$, Takashi Terauchi ${ }^{1}$, Mitsuru Koizumi ${ }^{1}$ and
} Masahisa Onoguchi $i^{*}$

\begin{abstract}
Background: Two novel methods of image reconstruction, xSPECT Quant (xQ) and XSPECT Bone (xB), that use an ordered subset conjugate gradient minimizer (OSCGM) for SPECT/CT reconstruction have been proposed. The present study compares the performance characteristics of $\times Q, \times B$, and conventional Flash3D (F3D) reconstruction using images derived from phantoms and patients.

Methods: A custom-designed body phantom for bone SPECT was scanned using a Symbia Intevo (Siemens Healthineers), and reconstructed XSPECT images were evaluated. The phantom experiments proceeded twice with different activity concentrations and sphere sizes. A phantom with $28-\mathrm{mm}$ spheres containing a ${ }^{99 \mathrm{~m}} \mathrm{Tc}$-background and tumor-to-normal bone ratios (TBR) of 1, 2, 4, and 10 were generated, and convergence property against various TBR was evaluated across 96 iterations. A phantom with four spheres (13-, 17-, 22-, and 28-mm diameters), containing a ${ }^{99 m}$ Tc-background at TBR4, was also generated. The full width at half maximum of an imaged spinous process $(10 \mathrm{~mm})$, coefficients of variance (CV), contrast-to-noise ratio (CNR), and recovery coefficients (RC) were evaluated after reconstructing images of a spine using Flash $3 \mathrm{D}(\mathrm{F} 3 \mathrm{D}), \mathrm{xQ}$, and $\mathrm{xB}$. We retrospectively analyzed images from 20 patients with suspected bone metastases (male, $n=13$ ) which were acquired using $\left[{ }^{99 \mathrm{~m}} \mathrm{~T} c\right] \mathrm{T} \mathrm{c}-$ (H)MDP SPECT/CT, then CV and standardized uptake values (SUV) at the $4^{\text {th }}$ vertebral body (L4) were compared after $\times \mathrm{Q}$ and $\mathrm{XB}$ reconstruction in a clinical setup.

Results: Mean activity concentrations with various TBR converged according to increasing numbers of iterations. The spatial resolution of $\times B$ was considerably superior to $\times Q$ and F3D, and it approached almost the actual size regardless of the iteration numbers during reconstruction. The $C V$ and $R C$ were better for $x Q$ and $x B$ than for F3D. The CNR peaked at 24 iterations for $\times Q$ and 48 iterations for $F 3 D$ and $\times B$, respectively. The $R C$ between $x Q$ and $\times B$ significantly differed at lower numbers of iterations but were almost equivalent at higher numbers of iterations. The reconstructed $\times Q$ and $x B$ images of the clinical patients showed a significant difference in the SUV $\max$ and $S U V_{\text {peak. }}$.

(Continued on next page)
\end{abstract}

\footnotetext{
* Correspondence: onoguchi@staff.kanazawa-u.ac.jp

Noriaki Miyaji is the first author.

${ }^{2}$ Department of Quantum Medical Technology, Institute of Medical

Pharmaceutical and Health Sciences, Kanazawa University, 5-11-80

Kodatsuno, Kanazawa, Ishikawa 920-0942, Japan

Full list of author information is available at the end of the article
}

\section{Springer Open}

(c) The Author(s). 2020 Open Access This article is licensed under a Creative Commons Attribution 4.0 International License, which permits use, sharing, adaptation, distribution and reproduction in any medium or format, as long as you give appropriate credit to the original author(s) and the source, provide a link to the Creative Commons licence, and indicate if changes were made. The images or other third party material in this article are included in the article's Creative Commons licence, unless indicated otherwise in a credit line to the material. If material is not included in the article's Creative Commons licence and your intended use is not permitted by statutory regulation or exceeds the permitted use, you will need to obtain permission directly from the copyright holder. To view a copy of this licence, visit http://creativecommons.org/licenses/by/4.0/. 


\begin{abstract}
(Continued from previous page)
Conclusions: The reconstructed $x Q$ and $x B$ images were more accurate than those reconstructed conventionally

using F3D. The $x B$ for bone SPECT imaging offered essentially unchanged spatial resolution even when the

numbers of iterations did not converge. The $\mathrm{XB}$ reconstruction further enhanced SPECT image quality using CT

data. Our findings provide important information for understanding the performance characteristics of the novel $x Q$ and $\mathrm{XB}$ algorithms.
\end{abstract}

Keywords: $x$ SPECT, Bone SPECT, OSCGM, Iteration number, Novel reconstruction

\section{Background}

Traditional bone imaging using ${ }^{99 \mathrm{~m}} \mathrm{Tc}$-labeled phosphate compounds is widely applied as diagnostic tools for detecting osseous metastases and staging malignant disease [1-3]. Hybrid bone imaging using single-photon emission computed tomography/computed tomography (SPECT/CT) can enhance image quality due to attenuation correction (AC), scatter correction (SC), and precisely localized tracer uptake. Römer et al. showed that $92 \%$ of indeterminate lesions could be correctly classified by SPECT/CT with a pronounced benefit for bone lesions [4]. Utsunomiya et al. also reported significantly improved diagnostic confidence for fused SPECT/CT image datasets compared with side-by-side views of images using both SPECT and CT modalities [5]. Hybrid SPECT/CT imaging in three dimensions (3D) has overcome the problem of planar bone imaging, which has high sensitivity, but low specificity, and thus improved the accuracy of diagnosing bone lesions [6, 7].

Recent advances in SPECT technology have included not only hardware but also software, such as image reconstruction algorithms. Absolute quantitation of ${ }^{99 \mathrm{~m}} \mathrm{Tc}$ bone SPECT/CT is becoming feasible as a diagnostic tool and as a means of monitoring treatment effects $[8$, 9]. Previous phantom and clinical studies have found that the quantitative accuracy of SPECT imaging using ${ }^{99 \mathrm{~m}} \mathrm{Tc}$ is within $\pm 10 \%[10,11]$. A multicenter study of four SPECT/CT systems also found that quantitative accuracy was maintained within $10 \%$ using $3 \mathrm{D}$ iterative reconstruction with $\mathrm{AC}, \mathrm{SC}$, and resolution recovery [12]. However, more reliable quantitative data are needed before quantitative bone SPECT imaging could become a standard clinical diagnostic procedure. Currently, the need to develop novel SPECT imaging techniques associated with absolute SPECT quantitation has been discussed in terms of cost, standardized uptake values (SUV), and dosimetry [13-15]. Quantitative SPECT/CT can overcome the downsides of positron emission tomography and has thus contributed to the rapid spread of quantitative nuclear medicine applications $[16,17]$.

Improved spatial resolution of SPECT images helps to improve the quantitation, detection, and precise localization of small lesions [18]. However, the spatial resolution of SPECT images remains poor. Tsui et al. suggested that multimodal image reconstruction would remarkably improve SPECT image quality [19]. Kuwert et al. also focused on quantitation and multimodal reconstruction as a methodological advance to further increase the value of bone SPECT/CT imaging [13]. The impact of using multimodal reconstruction methodology in SPECT imaging should be better quantifiability and excellent diagnostic confidence, although this awaits validation.

Siemens ${ }^{\circ}$ has introduced a technology called "xSPECT," which includes a novel iterative image reconstruction algorithm (ordered subset conjugate gradient minimizer; OSCGM) based on conventional ordered subset expectation maximization (OSEM; Flash 3D; F3D) to improve multimodal alignment in image space and thus enhance image quality. Onoguchi et al. described the differences between OSEM and OSCGM algorithms in detail [20]. Briefly, the XSPECT technology applies the Mighell merit function to suppress noise caused by the fast convergence of OSCGM reconstruction. Additionally, the National Institute of Standards and Technology (NIST) traceable calibration ${ }^{57} \mathrm{Co}$ point sources with 3\% uncertainty (99\% confidence level $(\mathrm{CI})$ ) were introduced by Siemens ${ }^{\oplus}$ to standardize quantitative ${ }^{99 \mathrm{~m}} \mathrm{Tc}$-SPECT. The SPECT voxel counts based on accurate correction can be converted to activity concentrations $(\mathrm{Bq} / \mathrm{mL})$ using a system planar sensitivity correction factor measured with a ${ }^{57} \mathrm{Co}$ source during reconstruction. This method of quantitative reconstruction is called "xSPECT Quant" $(x Q)$. Siemens ${ }^{\circ}$ also concurrently released bone-specific software with xSPECT features called "xSPECT Bone (xB)" [21], in which higherresolution CT data were added to enhance reconstructed images at tissue boundaries. Therefore, $\mathrm{xB}$ produces images of tracer distribution with far better resolution than F3D [22]. Some clinical reports have described that $x B$ bone SPECT images are more precise in terms of localization and offer better diagnostic confidence in staging malignant disease [23-25].

The fundamental theory of $\mathrm{xB}$ is that the application of image space information, divided into six tissue classes by higher-resolution $\mathrm{CT}$ data, minimizes interpolation errors in information obtained from anatomical modalities. Those reconstructed images have high spatial recognition due to denser spatial sampling. In contrast, $x Q$ applies a CT-derived reconstruction mask to reduce background 
noise [26]. A comparison of the two reconstruction methods revealed unexpected behavior of $\mathrm{xQ}$, which caused a decrease in the image quality of $>2$ subsets [27, $28]$. For both $x Q$ and $x B$, developers also found that noise is lower, and resolution is higher in 3- than 6-degree sampling [29]. Quantitative and physical indexes such as recovery coefficients (RC), SUV, and noise characteristics typically depend on image reconstruction and the reconstruction parameters. Although xSPECT imaging also depends on different reconstruction parameters, its impact has not yet been clarified. The present study aimed to determine the performance characteristics of the novel xSPECT algorithm. To our knowledge, this is the first attempt to clarify the functional differences between $x Q$ and $\mathrm{xB}$ based on phantom measurements and clinical data.

\section{Methods}

\section{Data acquisition and reconstruction}

All imaging data were acquired using a Symbia Intevo16 hybrid SPECT/CT system (Siemens Healthineers, Erlangen, Germany) comprising an integrated dual-head SPECT camera with a 16-slice helical CT scanner. We acquired SPECT images under the following parameters: $\pm 7.5 \%$ energy window at $140 \mathrm{keV}$ with a lower scatter window of $15 \%, 3 / 8$ " crystal thickness, low-energy high-resolution collimator, $256 \times 256$ matrix with $2.0-\mathrm{mm}$ pixels, and a total of 120 projections of $15 \mathrm{~s} /$ view over $360^{\circ}$ in a non-circular orbit continuous acquisition mode. Immediately following SPECT acquisition, CT images were acquired at $130 \mathrm{kV}$ and 70 ref $\mathrm{mA}$ using adaptive dose modulation (CARE Dose 4D; Siemens Healthineers) with a $512 \times 512$ matrix, pitch 1.5, 0.8-s rotation, and $2 \times 1.5-\mathrm{mm}$ collimation. The CT data were reconstructed at a $3.0-\mathrm{mm}$ slice thickness using a B31s attenuation filter (Siemens Healthineers).

We reconstructed the SPECT images using the algorithms $\mathrm{F} 3 \mathrm{D}, \mathrm{xQ}$, and $\mathrm{xB}$ and a 6-mm 3D Gaussian filter with various combinations of one fixed subset and 1-96 iterations. The OSEM-based F3D is equipped with depthdependent 3D resolution recovery using the Gaussian point-spread functions. The OSCGM-based $x Q$ and $x B$ are equipped with depth-dependent $3 \mathrm{D}$ resolution recovery using actual measured point-spread functions map over the entire FOV. The $\mathrm{xB}$ algorithm divides CT pixels into six tissue classes with smooth boundaries based on CT values or "zones" of air and lung, adipose, soft tissue, soft bone, cortical bone, metal material, and updates. The $\mathrm{xB}$ iterative operation can be weighted according to the corresponding zone class in the divided pixel; however, the iterative operation for each zone class based on the CT data does not increase the original count [21].

\section{Cross-calibration of SPECT imaging}

Counts from SPECT images reconstructed with F3D and xSPECT were converted to activity concentrations based on a cross-calibration factor (CCF) obtained from the relationship between the reconstructed counts and activity concentrations as well as system planar sensitivity, for quantitative comparisons.

In SPECT images using F3D, a circular region of interest (ROI) to measure SPECT count density (counts/mL) was placed at the center of the cylindrical phantom on the central slice and at \pm 1 and \pm 2 slices from the center. The CCF was automatically calculated using GIBONE software (Aze, Tokyo, Japan) as the ratio of the actual activity concentration (measured by the dose calibrator) in the phantom at the time of scanning to the measured SPECT count density per scan duration [30]. The dose calibrator used for cross-calibration was CRC15R (final calibration date by manufacturer: April 19, 2005). The dose calibrator was also confirmed and calibrated with a site-specific NIST-traceable ${ }^{68} \mathrm{Ge} /{ }^{68} \mathrm{Ga}$ source every 3 months [31, 32] (final calibration date in site: December 18, 2019). Therefore, we assume that the uncertainty of the measurement by the dose calibrator is small. The actual SUV was calculated as:

$$
\text { Calibration factor } \times \text { count density } /\left(\frac{\text { Injected activity }}{\text { Body weight (phantom volume })}\right)
$$

Reconstruction with $\mathrm{xQ}$ and $\mathrm{xB}$ precisely determines images in units of becquerel/milliliter that are converted using system planar sensitivity with an NIST traceable ${ }^{57}$ Co source [21]. The system planar sensitivity is a necessary parameter to allow for conversion between the count rate and units of absolute activity. This is defined as a measure of how many counts the gamma camera detects for every unit of activity in its field of view. Therefore, system planar sensitivity was measured with the traceable point source without scattering and attenuation to realize accurate and reproducible quantitation $[28,33]$. This source is recommended for all Siemens ${ }^{\circ}$ users to improve SPECT quantitation. It was automatically converted to the quantitative SPECT/CT data by the VB10 software (Siemens Healthineers).

\section{Phantom studies \\ Phantom design}

We custom-designed a physical three-dimensional phantom to determine the bone SPECT-specific distribution of activity and the linear attenuation coefficient (Fig. 1). This phantom can be used to generate SPECT images of bone metastasis with a realistic abdomen contour [34]. The phantom contains a ${ }^{99 \mathrm{~m}} \mathrm{Tc}$ solution to simulate soft tissue, the vertebral body, spinous and transverse process, and tumor region contained a bone-equivalent solution of $\mathrm{K}_{2} \mathrm{HPO}_{4}$ and ${ }^{99 \mathrm{~m}} \mathrm{Tc}$ [35]. The phantom experiments were conducted twice using different activity concentrations and sphere sizes as follows. Tumor, normal bone, and soft tissues in the phantom were 

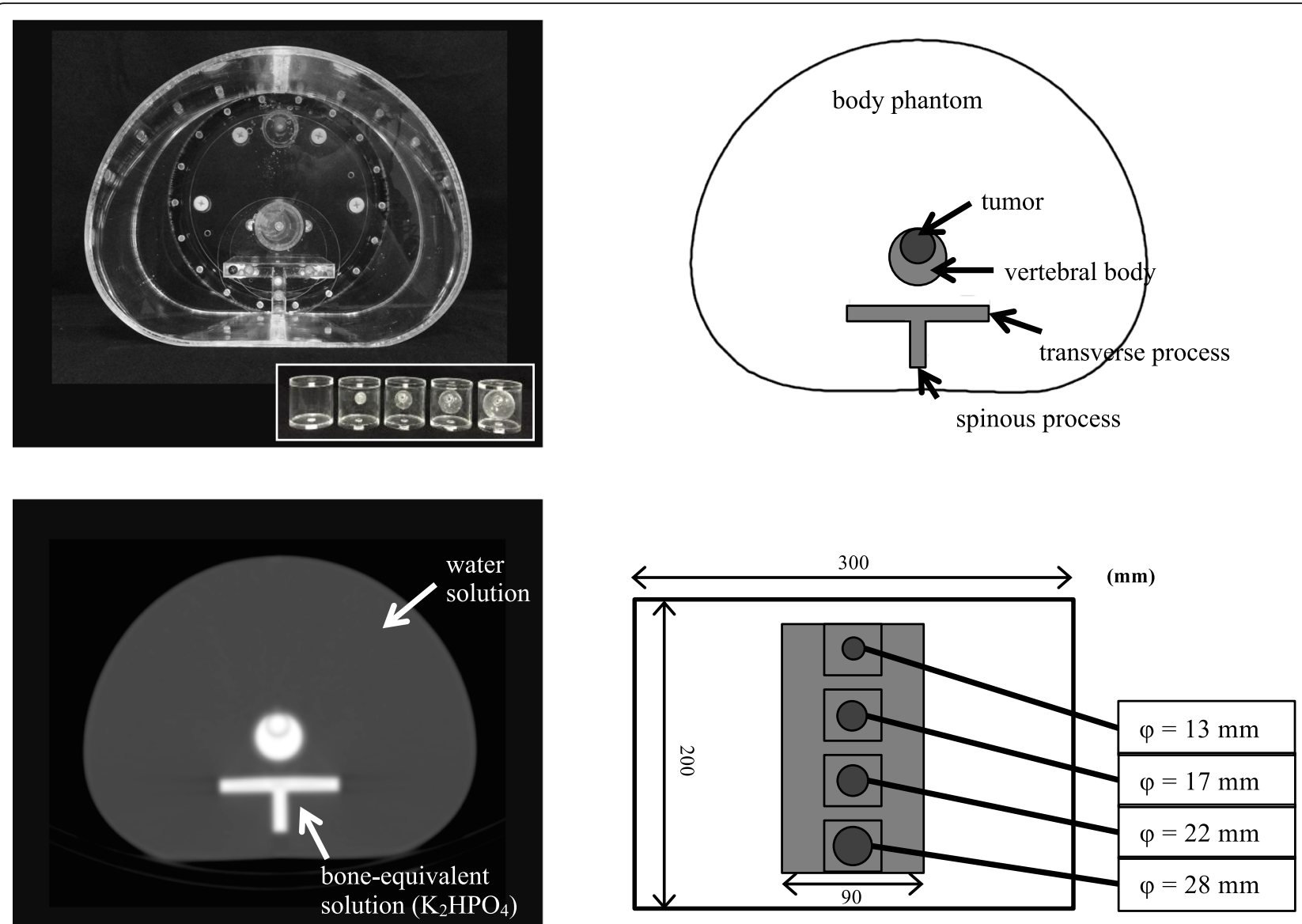

Fig. 1 Custom-designed phantom configured with vertebral body, spinous and transverse process, and a sphere set inside the vertebral body to simulate bone metastasis

immersed in a solution of ${ }^{99 \mathrm{~m}} \mathrm{Tc}$. In the first round of experiments, a body phantom with four $28-\mathrm{mm}$ diameter spheres was set and acquired at tumor-to-normal bone ratios (TBR) of $1,2,4$, and 10 at a normal bone activity level of $50 \mathrm{kBq} / \mathrm{mL}$. This phantom contained $8 \mathrm{kBq} / \mathrm{mL}$ of a ${ }^{99 m}$ Tc solution as the background activity of the soft tissue. That is, the boundary and the background do not differ at TBR1, but the difference in the activity concentration increases as a function of a higher TBR. We determined the activity concentrations of the simulated soft tissue, normal bone, and tumor at 8, 50, and 200 $\mathrm{kBq} / \mathrm{mL}$ (TBR4), respectively, in the second round of experiments using a phantom with 13-, 17-, 22-, 28-mmdiameter spheres.

\section{Data analysis}

The SPECT acquisition data in the first round of experiments were reconstructed using 1 subset and 1-96 iterations. We examined the effects of the reconstruction algorithms on various TBR in the $28-\mathrm{mm}$ sphere and then determined the optimal reconstruction parameters based on the result of convergence characteristics.
Phantom images containing simulated tumors of different sizes were continuously analyzed in terms of the spatial resolution of a $10-\mathrm{mm}$ spinous process, the coefficient of variance $(\mathrm{CV})$, the contrast-to-noise ratio (CNR) of the vertebral body, and $\mathrm{RC}$ as quantitative parameters. We drew profile curves on the spinous process and measured the full width at half maximum (FWHM). The CV was evaluated at an $80 \%$ circular ROI (ROI80\%) placed at the center of the vertebral body. In addition, a total of sixty ROI80\% including \pm 1 and \pm 2 slices were placed around the vertebral body to calculate as background CV. The $\mathrm{CV}$ was calculated as standard deviation (SD) divided by mean in the ROI. The CNR and $\mathrm{RC}$ at each sphere were determined by setting circular ROIs with diameters of $13,17,22$, and $28 \mathrm{~mm}$. The CNR at TBR 4 was calculated as $(\mathrm{Hs}-\mathrm{Hnb}) / \sigma n b$, where Hs and $\mathrm{Hnb}$ are the activity concentrations measured in the spheres and normal bone, respectively, and onb is the voxel $\mathrm{SD}$ in the normal bone. The $\mathrm{RC}$ was defined as the ratio of the SPECT-based and the true activity concentration $(\mathrm{kBq} / \mathrm{mL})$ for each sphere. 


\section{Clinical study Imaging protocol}

We analyzed data from 20 consecutive patients who had undergone bone SPECT/CT imaging for metastatic prostate or breast cancer (male, $n=13$; female, $n=7$; median age, 62 years; range, $40-83$ years; average weight, $65.2 \pm 13.4 \mathrm{~kg}$; range, $51.8-78.6 \mathrm{~kg}$ ). The optimal parameters of the convergence characteristic in the phantom study were applied to the clinical reconstruction condition in $\mathrm{xQ}$ and $\mathrm{xB}$. Bone SPECT/CT imaging proceeded from the abdomen to the pelvis $\sim 2.5-4 \mathrm{~h}$ after delivering an intravenous injection of $1003.4 \pm 102.8 \mathrm{MBq}$ ${ }^{99 \mathrm{~m}}$ Tc-methylene diphosphonate ([$\left.{ }^{99 \mathrm{~m}} \mathrm{Tc}\right] \mathrm{Tc}-\mathrm{MDP}$; FUJIFILM Toyama Chemical, Tokyo, Japan) or hydroxymethylene diphosphonate ([ $\left.{ }^{99 \mathrm{~m}} \mathrm{Tc}\right] \mathrm{Tc}-\mathrm{HMDP} ;$ Nihon Medi-Physics, Tokyo, Japan). The average amount of injected ${ }^{99 \mathrm{~m}}$ Tc was $15.9 \pm 2.8$ (range, 13.1-18.7) $\mathrm{MBq} /$ $\mathrm{kg}$. The Ethics Committee at the Cancer Institute Hospital of JFCR approved this clinical study (approval no. 2015-1151). These clinical data were retrospectively analyzed, and the results did not influence any further therapeutic decision-making.

\section{Data analysis}

The noise characteristics and quantitative performance of the clinical SPECT image were analyzed at the level of the 4th vertebral body (L4) [36]. We adjusted and placed a ROI of $80 \%$ size on the center of the axial slice in the section after measured the ROI of the vertebral body guided by the CT boundaries of the fused SPECT/CT images. We normalized the $\mathrm{SUV}_{\text {max }}, \mathrm{SUV}_{\text {mean }}$, and SUVpeak by the weight of each patient. An average and maximum concentration in milliliter within a ROI would produce an estimate of the SUV, which is defined here as $S U V_{\text {mean }}$ and $S U V_{\text {max }}$, respectively. $S U V_{\text {peak }}$ has been suggested as an alternative to $\mathrm{SUV}_{\text {max }}$. SUV peak within a setting ROI is an average SUV calculated within a fixed size (this is a sphere with a diameter of approximately $1.2 \mathrm{~cm}$ to require a $1-\mathrm{cm}^{3}$ volume spheric ROI), placed highest uptake region including maximum pixel value. Because this VOI encompasses several pixels, $\mathrm{SUV}_{\text {peak }}$ is assumed to be less affected by image noise than $\mathrm{SUV}_{\max }$. These data were analyzed using PETSTAT software (AdIn Research, Tokyo, Japan) (Fig. 2).

\section{Statistical analysis}

All SUV and CV indices in the $\mathrm{xQ}$ and $\mathrm{xB}$ groups were compared using Wilcoxon signed-rank tests after evaluating the non-normal distribution using KolmogorovSmirnov tests. Values were considered statistically significant when $P<0.05$. These data were statistically analyzed using SPSS Statistics software (IBM Corp., Armonk, NY, USA).
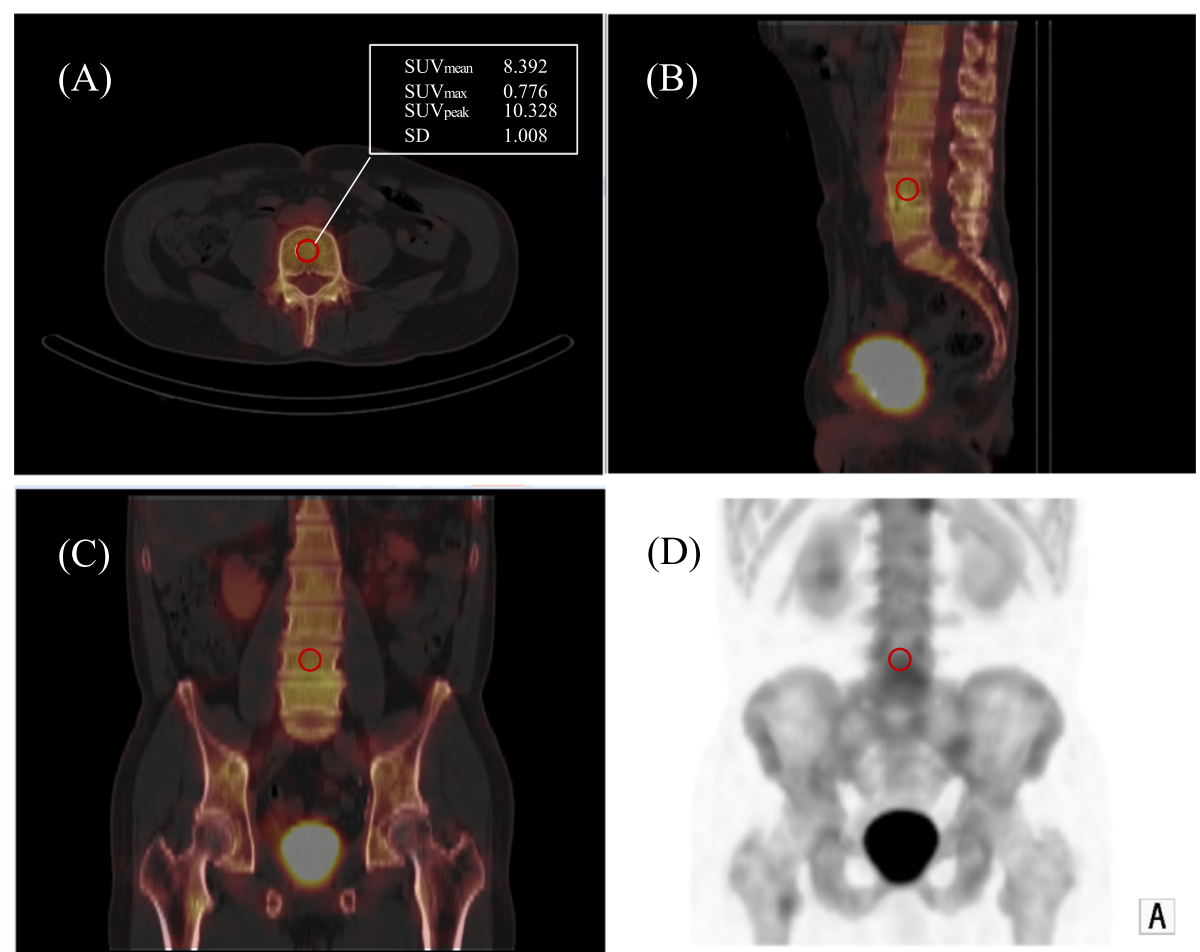

(D)

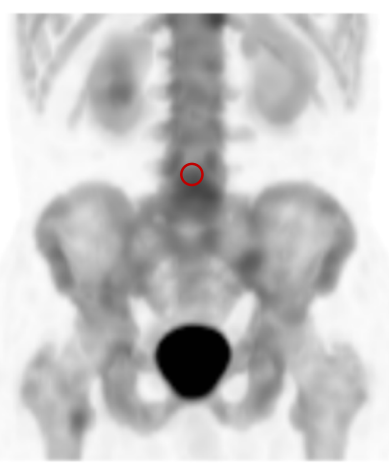

A

Fig. 2 We set ROI80\% (red circle) at the center of xB imaged based on a fused axial image, then adjusted by sagittal and coronal images. a Fused axial image. b Fused sagittal image. c Fused coronal image. d MIP image 


\section{Results}

Phantom studies

\section{Convergence for various TBR}

Figure 3 shows the SPECT data reconstructed using between 1 and 96 iterations. Regardless of the reconstruction model and iteration number, the means were better than the maximum activity concentrations for the two lowest TBR values (Fig. 3a, b), whereas those of the maximum activity concentrations were better results for the highest TBR values (Fig. 3c, d). In Fig. 3d, the maximum activity concentrations were the highest with F3D and better than those for both $\mathrm{xQ}$ and $\mathrm{xB}$. On the other hand, the maximum activity concentration with XSPECT did not converge and increased in proportion to the iteration numbers. The mean activity concentration converged with increasing iterations regardless of the TBR. The mean activity concentrations of $\mathrm{xQ}$ and $\mathrm{xB}$ were essentially equivalent at $>24$ iterations. The mean activity concentration was lower for F3D than $\mathrm{xQ}$ and $\mathrm{xB}$.

\section{Spatial resolution}

Figure 4 shows the spatial resolution of the spinous process for various iterations. The FWHM with $\mathrm{xQ}$ and F3D considerably improved when the iteration number increased, but the spatial resolution produced by the $\mathrm{xB}$ algorithm was optimal. The FWHM of the $x Q$ and F3D reconstructions converged at about 15 and $20 \mathrm{~mm}$, respectively. In contrast, the $\mathrm{xB}$ values remained similar to the actual size $(10 \mathrm{~mm})$ regardless of iteration numbers. Figure 5 shows the results of the xSPECT and F3D images with 1 subset and F3D images with 3 subsets at 48 iterations, respectively. The boundary of the vertebral body was visually indistinct on reconstructed F3D and $x Q$ bone SPECT images, whereas it was clearly visible in the reconstructed $\mathrm{xB}$ images. In terms of background region, $\mathrm{xB}$ and $\mathrm{F} 3 \mathrm{D}$ images with 1 subset produced clearer images than $x Q$. Also, the $x Q$ with 1 subset and F3D with 3 subsets respectively were visually equivalent level.

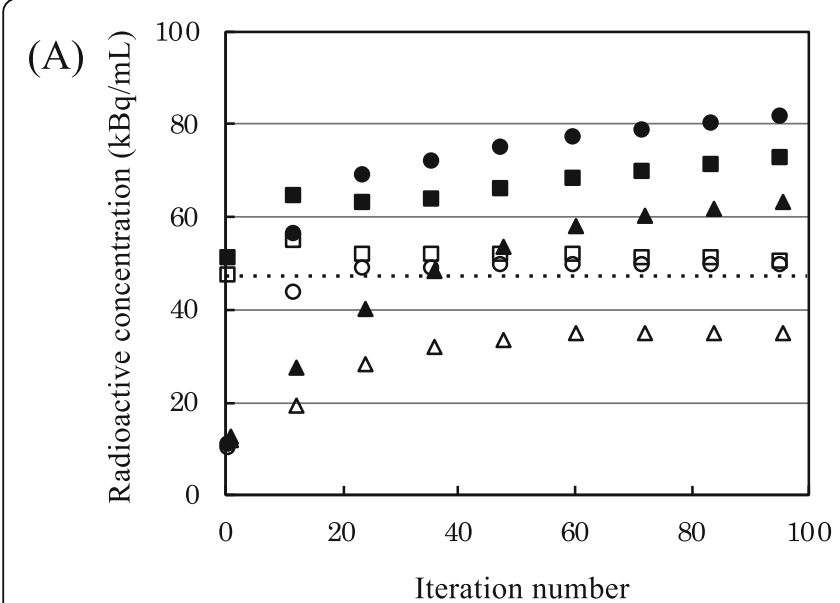

(C)

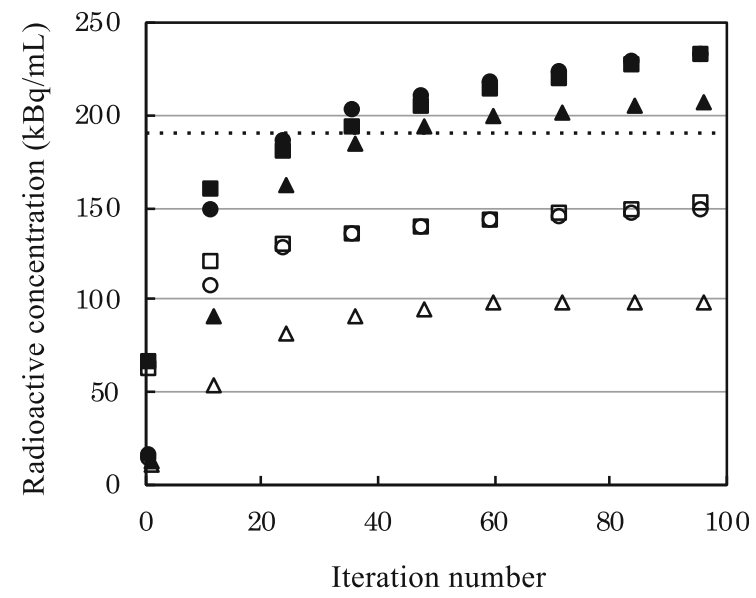

(B)

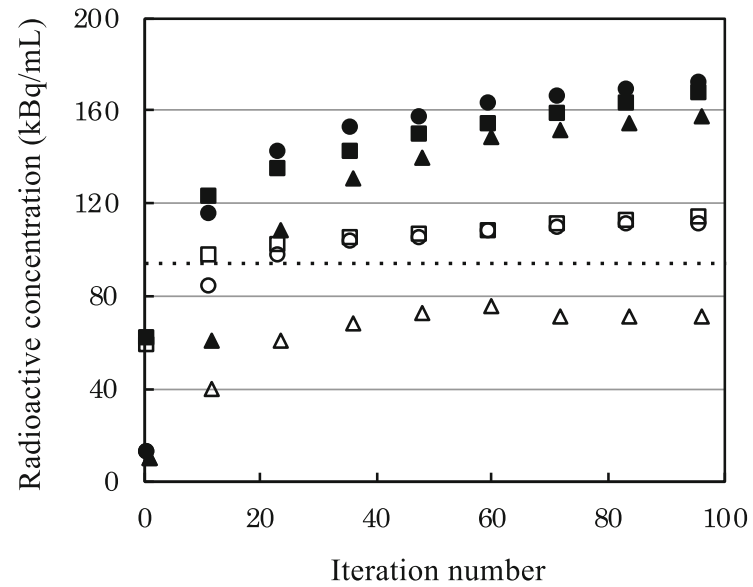

(D)

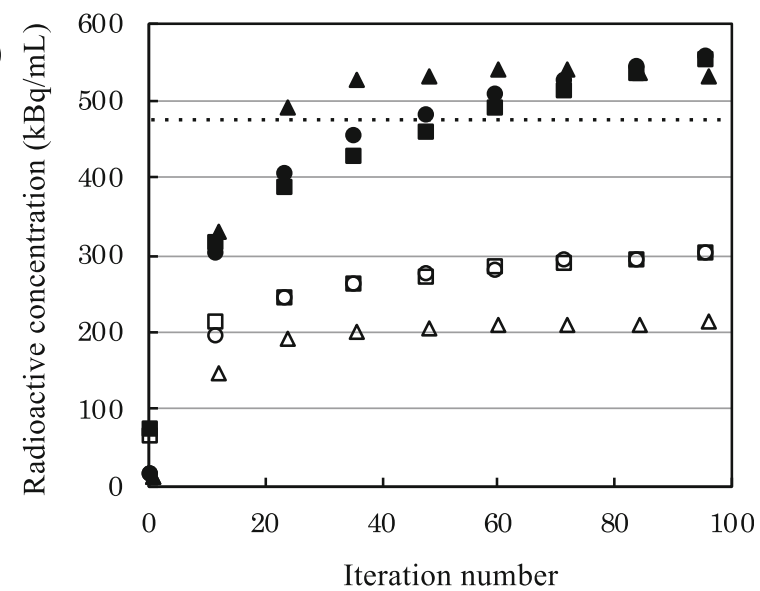

Fig. 3 Reconstruction plots showing quantitative distribution in TBR1 (a), 2 (b), 4 (c), and 10 (d). The filled and unfilled symbols indicate maximum and mean activity concentrations, respectively. The dotted line is the actual activity concentration of phantom. Filled and unfilled triangles indicate Flash 3D (F3D). Filled and unfilled circles indicate xSPECT Quant (xQ). Filled and unfilled squares indicate xSPECT Bone (xB) 

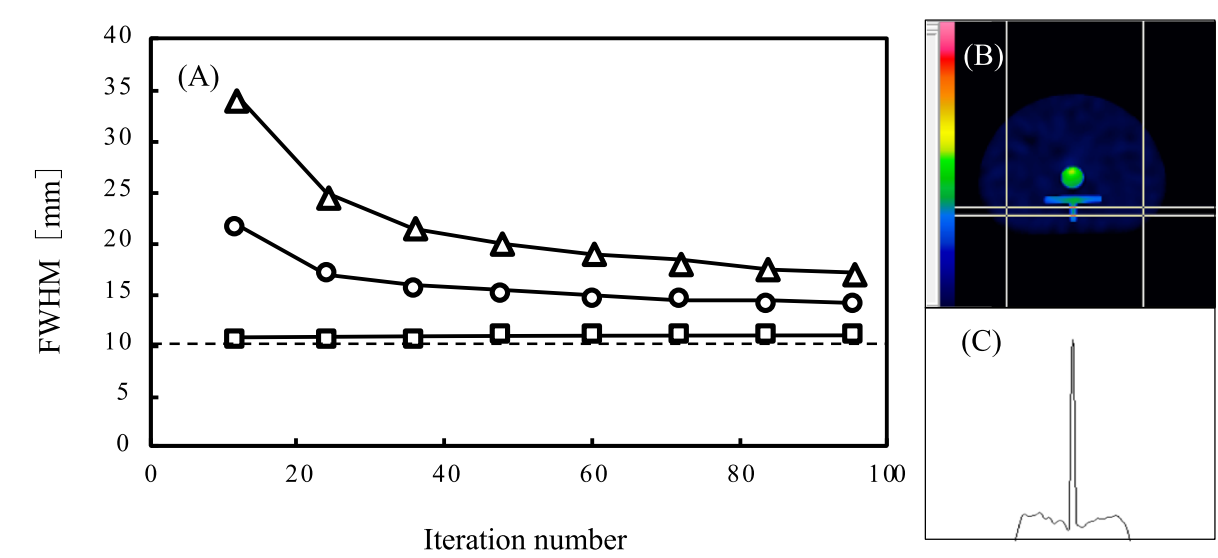

Fig. 4 The FWHM measurement shown by the profile curve on the spinous process cross-section. a Spatial resolution of three reconstructions at various iterations. The dotted line is the actual size of the phantom. Unfilled triangle indicates Flash 3D (F3D). Unfilled circle indicates XSPECT Quant (XQ). Unfilled square indicates XSPECT bone (XB). b A sample measurement of an XB image at 12 iteration numbers. c A measurement profile of an $\times B$ image at 12 iteration numbers. The plateau signal around the spinous process is a distribution of background region in the phantom

\section{Noise characteristics}

Figure 6 shows the CV of the vertebral body and background region according to the number of iterations, respectively. The CV in the vertebral body was higher in F3D than in $x Q$ and $x B$ as the iteration numbers increased, and the amount of noise was similar between $\mathrm{xQ}$ and $\mathrm{xB}$. Those of $\mathrm{xQ}$ and $\mathrm{xB}$ at $>24$ iterations were both relatively stable at 0.2 . On the other hand, the background CV of $\mathrm{xQ}$ significantly was inferior to other reconstruction. The $\mathrm{CV}$ of $\mathrm{xB}$ and $\mathrm{F} 3 \mathrm{D}$ showed an equivalent value at 48 iteration numbers. Figure 7 shows the CNR in the vertebral body region according to the iteration numbers. The mean and max CNR were similar for each reconstruction. Although the mean CNR was better in the order of $\mathrm{xB}, \mathrm{xQ}$, and $\mathrm{F} 3 \mathrm{D}$ as the iteration numbers increased, the CNR of F3D and $\mathrm{xB}$ at $>48$, and $\mathrm{xQ}$ at $>24$ iterations decreased.

\section{Recovery coefficient}

Figure 8 shows the RC of the vertebral body for 12-96 iterations. The RC in all algorithms improved with increasing sphere size. The RC was relatively higher with $\mathrm{xB}$ than with the other algorithms at 12 iterations, and the differences in the $\mathrm{RC}$ between $\mathrm{xQ}$ and $\mathrm{xB}$ were essentially equivalent as a function of the increasing numbers of iterations. The $\mathrm{RC}$ was lower for F3D than $\mathrm{xQ}$ and $\mathrm{xB}$ at the same number of iterations, but the $\mathrm{RC}$ of F3D after 36 iterations was better than that of $x Q$ after 12 (Fig. 8g).

\section{Clinical study}

Table 1 shows the $\mathrm{SUV}_{\text {max }}, \mathrm{SUV}_{\text {peak }}, \mathrm{SUV}_{\text {mean }}$, and CV under clinical conditions. The quantitative SPECT values were much higher for some patients. The statistical findings showed a significant difference in the $\mathrm{SUV}_{\max }$ and $\mathrm{SUV}_{\text {peak }}$ between $\mathrm{xQ}$ and $\mathrm{xB}$. However, $\mathrm{SUV}_{\text {mean }}$ and CV on SPECT images reconstructed with $\mathrm{xQ}$ and $\mathrm{xB}$ did not significantly differ $(P>0.05)$.

\section{Discussion}

We validated novel xSPECT and conventional F3D reconstruction algorithms using experimental data derived from phantoms. Differences between $\mathrm{xB}$ and $\mathrm{xQ}$ were quantified based on clinical data from patients. The phantom study found image quality and quantitative accuracy of $\mathrm{xSPECT}$ were considerably superior to those

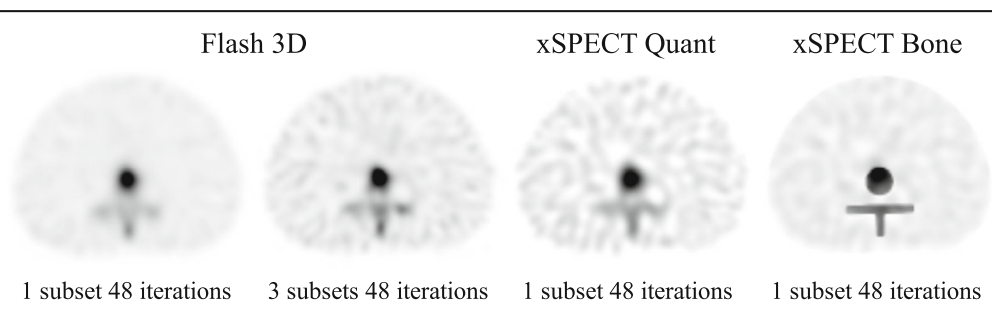

Fig. 5 Representative transaxial images of SPECT datasets including three reconstructions at TBR4. Reconstructed images showed the xSPECT and F3D images with 1 subset and with 3 subsets at 48 iterations, respectively 


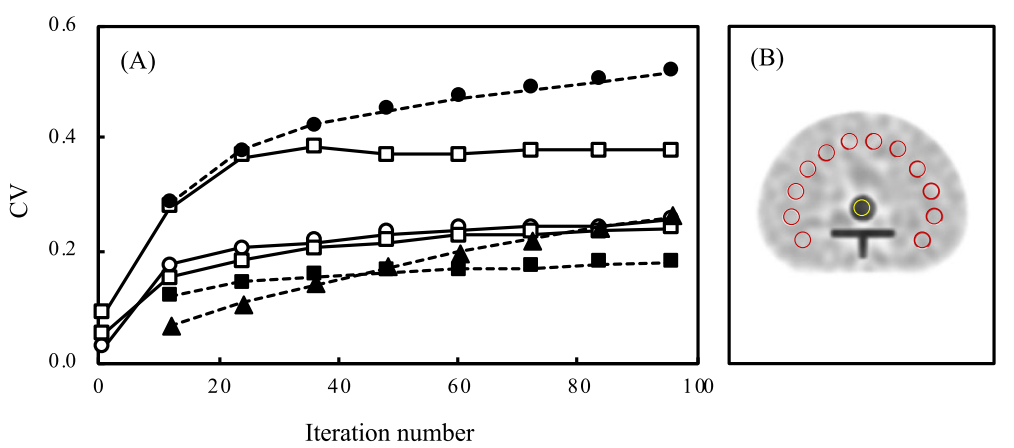

Fig. 6 The coefficient of variance (CV) measurement in the ROI placed at the center of the vertebral body. a The CV as a function of iteration numbers. The black line is the CV of the vertebral body and the dot line in black is the background CV, respectively. Filled and unfilled triangles indicate Flash 3D (F3D). Filled and unfilled circles indicate XSPECT Quant (XQ). Filled and unfilled square xSPECT Bone (xB). b A sample measurement of an $\times B$ image at 1 subset and 48 iterations

of F3D. However, background noise obviously differed visually for $\mathrm{xQ}$ without weighted correction compared with F3D due to increasing noise caused by fast convergence. We also found that the high spatial resolution of $\mathrm{xB}$ was maintained regardless of the iteration numbers. The $S U V_{\max }$ and the $\mathrm{SUV}_{\text {peak }}$ in the clinical study significantly differed between $\mathrm{xQ}$ and $\mathrm{xB}$; thus, we concluded that $\mathrm{xB}$ could serve as an essential diagnostic tool for bone SPECT imaging in terms of quantitative accuracy and spatial resolution.

Regardless of the reconstruction models, the maximum activity concentration in the TBR1 and TBR2 spheres was overestimated compared with actual activity concentration (Fig. 3). This can be explained by the fact that the maximum activity concentration in the sphere theoretically increased because of increasing statistical noise at lower counts [37]. The activity is pushed into the contours of the sphere due to the spatial constraint by a CT-based intensity masking for OSCGM. Tran-Gia et al. showed the mean $x Q$ inside the sphere remained relatively similar to F3D; the distribution in the profile curve was drastically changed compared to F3D. The maximum in the sphere center was increasing, while the edge was decreasing [27]. Therefore, no convergence was reached for the $\mathrm{SUV}_{\max }$ of xSPECT in Fig. 3, and the recovery of xSPECT was highly dependent on the iteration number. In contrast, F3D for higher TBRs is independent of the total iteration numbers; the maximum activity concentration of the F3D exceeded those of xSPECT (Fig. 3d). The merit function incorporated in xSPECT might enhance noise suppression as a function of higher activity concentration. However, this effect reduced for higher iteration numbers because xSPECT does not converge. The xSPECT reconstruction has several unknown features, so this is only one potential explanation. On the other side, the mean activity concentration approached the actual activity concentration at lower TBR. When the activity concentrations of tumor and normal bone were equal $(\mathrm{TBR}=1)$, spill-out by partial volume did not occur because the activity concentrations inside and outside the ROI were almost equivalent. TBR 1 was slightly overestimated due to the activity concentration being increased by the statistical noise. At a higher TBR, the mean activity concentration
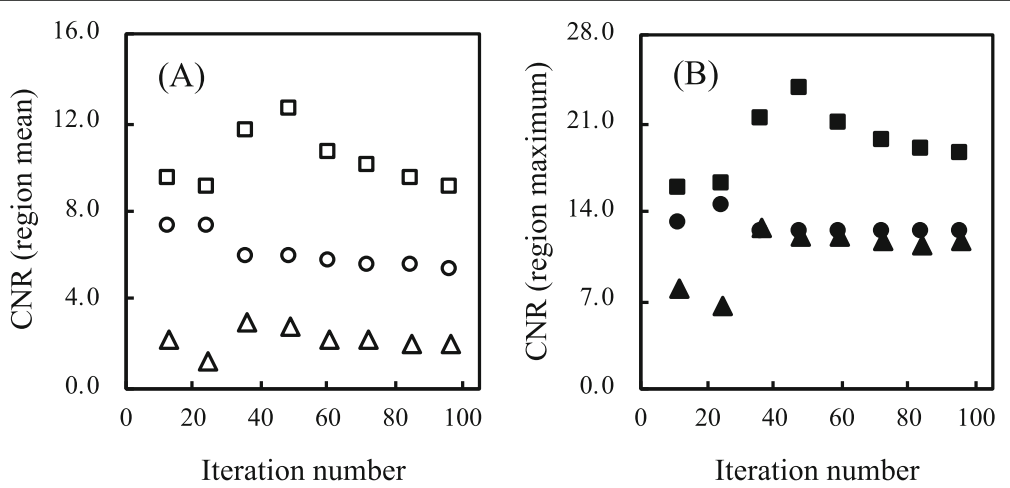

Fig. 7 The contrast-to-noise ratio (CNR) measured by activity concentrations for the hot spheres and normal bone at TBR 4. a The mean CNR as a function of iteration numbers. $\mathbf{b}$ The maximum CNR as a function of iteration numbers. Filled and unfilled triangles indicate Flash 3D (F3D). Filled and unfilled circles indicate XSPECT Quant (XQ). Filled and unfilled square indicate XSPECT Bone (xB) 
(A)

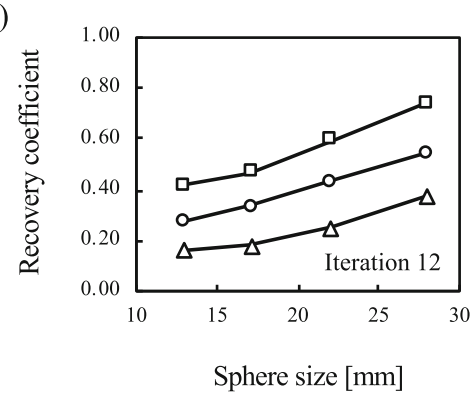

(D)

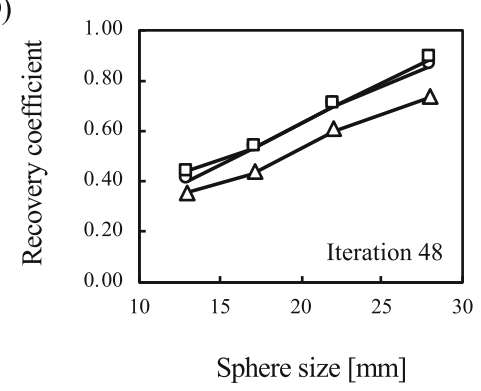

(G)

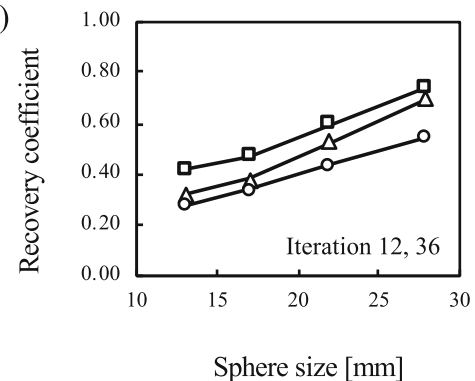

(B)

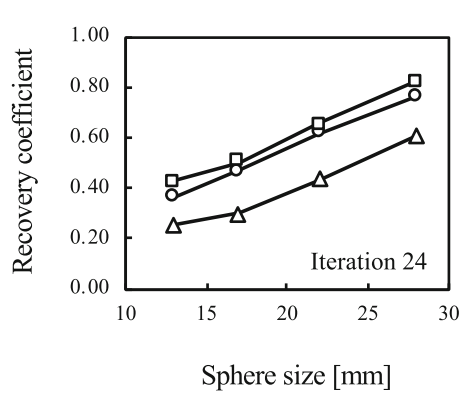

(E)

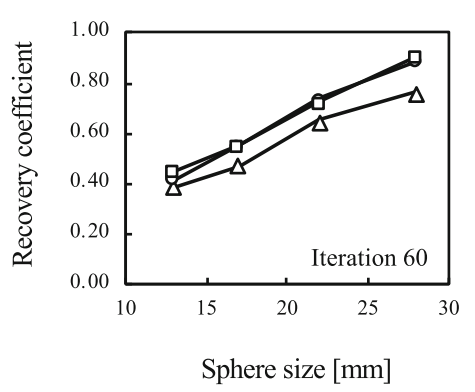

(H)

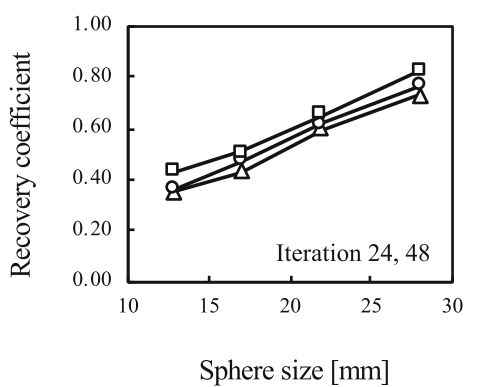

(C)

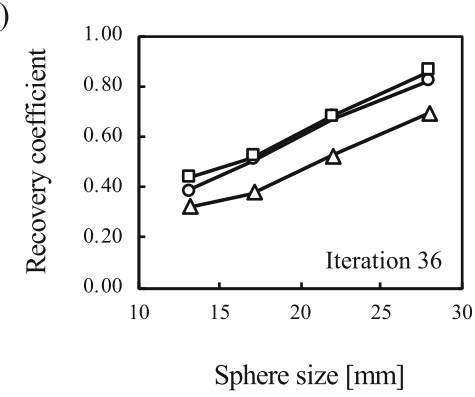

(F)

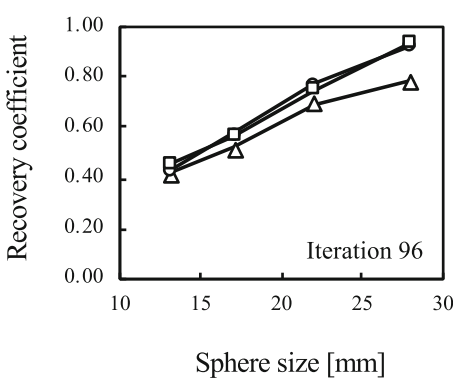

Fig. 8 Recovery coefficients of three reconstructions at various numbers of iterations. The numbers of iterations in $\mathbf{a}$, $\mathbf{b}, \mathbf{c}$, $\mathbf{d}$, e, and $\mathbf{f}$ are 12,24 , $36,48,60$, and 96 , respectively. In addition, the numbers of iterations in $\mathbf{g}$ and $\mathbf{h}$ show the different parameters of 36 and 48 in Flash $3 \mathrm{D}$ and 12 and 24 in XSPECT, respectively. Unfilled triangle indicates Flash 3D. Unfilled circle indicates xSPECT Quant. Unfilled square indicates XSPECT Bone

was underestimated due to spillage from the sphere into the background [38]. The quantitative differences between F3D and xSPECT are influenced by statistical noise based on convergence and by partial volume effects caused by lower spatial resolution. Our results showed that the mean activity concentrations for F3D essentially converged within 48 iterations, but those for $\mathrm{xQ}$ and $\mathrm{xB}$ similarly converged at $>24$ iterations. The FWHM for the $x Q$ after 36 iterations converged, and the $\mathrm{RC}$ between the $\mathrm{xB}$ and $\mathrm{xQ}$ was almost equivalent at over 36 iterations. The maximum activity concentrations with xSPECT did not converge even for high iteration

Table 1 Values for SUV mean, SUV max $S_{\text {SUV }}$ peak, and CV of the 4th vertebral body in $x Q$ and $x B$ reconstructions

\begin{tabular}{|c|c|c|c|c|}
\hline Quantitative indices & Reconstruction type & Mean \pm SD & Min-max & $P$ value \\
\hline \multirow[t]{2}{*}{$S U V_{\text {mean }}$} & $x Q$ & $7.10 \pm 5.81$ & $2.50-30.34$ & \multirow[t]{2}{*}{0.084} \\
\hline & $x B$ & $7.03 \pm 5.91$ & $2.29-30.76$ & \\
\hline \multirow[t]{2}{*}{$S U V_{\max }$} & $\mathrm{xQ}$ & $12.29 \pm 11.84$ & $4.87-58.16$ & \multirow[t]{2}{*}{0.021} \\
\hline & $x B$ & $11.90 \pm 12.16$ & $4.67-59.07$ & \\
\hline \multirow[t]{2}{*}{$S U V_{\text {peak }}$} & $\mathrm{xQ}$ & $11.63 \pm 11.25$ & $4.68-55.73$ & \multirow[t]{2}{*}{0.001} \\
\hline & $x B$ & $11.24 \pm 11.37$ & $4.56-55.52$ & \\
\hline \multirow[t]{2}{*}{ CV } & $x Q$ & $0.28 \pm 0.17$ & $0.13-0.80$ & \multirow[t]{2}{*}{0.141} \\
\hline & $x B$ & $0.26 \pm 0.17$ & $0.12-0.69$ & \\
\hline
\end{tabular}


numbers as shown in Fig. 3. The iteration numbers are associated with a trade-off between signal and noise. Considering the increase in noise, we determined that 30 iterations were the most appropriate for XSPECT reconstruction in clinical practice.

The FWHM of F3D after > 36 iterations was better than $\mathrm{xQ}$ after 12 iterations that have not fully converged; however, the FWHM with $x Q$ and F3D considerably improved and fully converged at $\sim 15$ and $20 \mathrm{~mm}$, respectively, at a high number of iterations. Therefore, image quality was better for $\mathrm{xQ}$ than F3D at the appropriate parameter. In contrast, the $\mathrm{xB}$ algorithm divided into zone class generated unique results, unlike the observed $x Q$. The spatial resolution for $x B$ remained almost unchanged even for lower iteration numbers, and the actual size of $10 \mathrm{~mm}$ was almost achieved. The zone class of each tissue was based on high-resolution CT images with delineated edges; therefore, the FWHM of $\mathrm{xB}$ reflects the relationship to CT resolution. Additionally, the $\mathrm{xB}$ iterative operation is weighted by zero or other value according to the corresponding zone class in the divided pixel [21]. We considered that not only bone classes weighted by the optimal value, but also non-bone classes weighted by zero with a zonal map were responsible for the improved spatial resolution using the $\mathrm{xB}$ technology.

The xSPECT can compensate for SPECT images by applying the merit function in the higher noise caused by the faster convergence of OSCGM reconstruction. This method of reconstruction adopts the Mighellmodified chi-squared gamma statistic algorithm. Shinohara et al. indicated that Mighell-modified noise suppression was better than other image reconstructions based on chi-square statistics [39]. The CV of $x Q$ and $\mathrm{xB}$ did not exceed that of F3D at 12 iterations regardless of the iteration numbers. Thus, xSPECT with the Mighell-modified merit function considerably suppressed noise compared with F3D algorithms at the same number of iterations. For one subset of reconstructed images, a more apparent problem is the increasing image noise in the background region and in hot spheres according to the iteration numbers. The background noise in the $\mathrm{xQ}$ image rapidly increased and significantly was inferior to the other reconstructed images at 48 iterations (Fig. 5 and Fig. 6). However, Armstrong et al. reported that the greatest $\mathrm{CNR}$ for $\mathrm{xQ}$ is achieved at 48 iterations for one subset [28]. Our findings indicated that the greatest CNR for $x Q$ was at 24 iterations and that the RC was higher than for F3D at 48 iterations (Figs. 7 and $8 \mathrm{~h}$ ). On the other hand, $\mathrm{xB}$ suppressed image noise more effectively than F3D and $x Q$ (Fig. 6). Because $\mathrm{xB}$ reconstruction has weighted correction for every zone class, the impact of noise suppression differed between $\mathrm{xQ}$ and $\mathrm{xB}[40]$. The $\mathrm{CNR}$ in $\mathrm{xB}$ reached the maximum at 48 iterations, and noise suppression decreased in $\mathrm{xB}$ at $>48$ iterations. In regions with inadequate uptake such as soft tissues, the $\mathrm{xQ}$ based on the OSCGM algorithm might lead not only to an increased $\mathrm{CV}$ according to iteration setting, but also to ramifications for lesion detectability. Therefore, the $\mathrm{xQ}$ requires further careful optimization of the iteration numbers than F3D and $x B$.

The present study assessed data from 20 patients with suspected bone metastases. The SPECT values of measured L4 had a wide SUV range because some patients had various pathologies (bone metastasis $(n=7)$, degenerative $(n=5)$, and trauma $(n=3))$. Our clinical study found a significant difference in $S U V_{\text {peak }}$ and $S U V_{\max }$, and this quantitative difference between $x Q$ and $x B$ could be interpreted as noise suppression owing to weighted correction based on zone map system. Because the $\mathrm{SUV}_{\text {peak }}$ is less susceptible to statistical noise compared with $\mathrm{SUV}_{\max }$ [37], it significantly differed between $\mathrm{xQ}$ and $\mathrm{xB}(p=0.001)$. The clinical $\mathrm{xB}$ image with high resolution can not only reveal bone microlesions but also improve diagnostic confidence [23]. Therefore, we considered that clinical evaluation for $\mathrm{xB}$ images with $\mathrm{SUV}_{\text {peak }}$ could provide more accurate and reliable diagnostics. To calculate $\mathrm{SUV}_{\text {peak }}$ entails expressing the maximum average voxel value within a spherical volume of $1 \mathrm{~cm}^{3}$, but the $\mathrm{xB}$ is useful to enhance diagnostics for bone SPECT images in terms of quantitative and qualitative superiority. However, the quantitative variation caused by misalignments such as motion and respiratory errors during clinical scanning is a concern. Reconstruction using the $\mathrm{xB}$ algorithm might behave differently due to the unique zone map system. Therefore, misalignment between SPECT and CT images due to respiratory errors such as those caused by the ribs and sternum should be considered when clinically applying $\mathrm{xB}$.

The present study has several limitations. The reconstructed SPECT images were assessed using different cross-calibration methods. The CCF on quantitative SPECT images varied depending on the activity concentration [41]. Thus, slight quantitative errors might arise between the F3D and xSPECT models. In addition, the body type of the 20 patients and the amounts of injected tracer were essentially standard (average, $15.9 \pm 2.8$ $\mathrm{MBq} / \mathrm{kg}$ ). We could not consider dependence on physique into consideration, and the effects of factors such as counts and scattering remain unclear. Further study is required to assess the relationship between body type and the quality of images reconstructed using the xSPECT algorithm.

\section{Conclusions}

Bone images were qualitatively and quantitatively improved when reconstructed using OSCGM-based $\mathrm{xSPECT}(\mathrm{xQ}$ and $\mathrm{xB})$ compared with the OSEM-based 
F3D reconstruction. The quality of images under optimized $\mathrm{xB}$ reconstruction conditions was better because of sharper demarcation and lower background noise. One unique aspect of the bone structures in $\mathrm{xB}$ reconstructions is that the image content such as spatial resolution was independent of the iteration numbers. Our findings provide important information that should facilitate understanding of the performance characteristics of the novel $\mathrm{xQ}$ and $\mathrm{xB}$ algorithms.

\section{Abbreviations}

XQ: xSPECT Quant; xB: XSPECT Bone; OSCGM: Ordered subset conjugate gradient minimizer; F3D: Flash 3D; FWHM: Full width at half maximum; CV: Coefficients of variance; RC: Recovery coefficients; SUV: Standardized uptake value; L4: 4th vertebral body; AC: Attenuation correction; SC: Scatter correction; SPECT/CT: Single-photon emission computed tomography/ computed tomography; OSEM: Ordered subset expectation maximization; CCF: Cross-calibration factor; TBR: Tumor-to-normal bone ratios; ROI: Regions of interest

\section{Acknowledgements}

We would like to thank the staff at the Diagnostic Imaging Center in Cancer Institute Hospital of JFCR. Parts of this manuscript were presented at the 2018 SNMMI Annual Meeting and the corresponding abstract published in JNM (J Nucl Med May 1, 2018 vol. 59 no. supplement 1 1792).

\section{Conflict of interest}

None

\section{Authors' contributions}

NM contributed to the study design, phantom data acquisition, and analysis of the data. KM and $\mathrm{MO}$ contributed to the study design, analysis of the data, and draft and critical revision of the manuscript. HI and AT contributed to the preparation of the study and critical revision of the manuscript. HI contributed to phantom data acquisition and interpretation. TT and MK contributed to the critical revision of the manuscript. All authors read and approved the final manuscript.

\section{Funding}

None

\section{Availability of data and materials}

All data generated or analyzed during this study are included in this published article.

\section{Ethics approval and consent to participate}

The Ethics Committee at the Cancer Institute Hospital of JFCR approved this clinical study (approval no. 2015-1151). The results of this retrospective study did not influence any further therapeutic decision-making.

\section{Consent for publication}

Not applicable

\section{Competing interests}

The authors declare that they have no competing interests

\section{Author details}

'Department of Nuclear Medicine, Cancer Institute Hospital of Japanese Foundation for Cancer Research, 3-8-31 Ariake, Koto-ku, Tokyo 135-8550, Japan. ${ }^{2}$ Department of Quantum Medical Technology, Institute of Medical Pharmaceutical and Health Sciences, Kanazawa University, 5-11-80 Kodatsuno, Kanazawa, Ishikawa 920-0942, Japan. ${ }^{3}$ Department of Radiological Sciences, School of Health Science, International University of Health and Welfare, 2600-1 Kitakanemaru, Ohtawara, Tochigi 324-8501, Japan. ${ }^{4}$ Department of Radiology, Toyohashi Municipal Hospital, 50, Aza Hachiken Nishi, Aotake-Cho, Toyohashi, Aichi 441-8570, Japan.
Received: 31 January 2020 Accepted: 10 June 2020

Published online: 29 June 2020

\section{References}

1. Krasnow AZ, Hellman RS, Timins ME, Collier BD, Anderson T, Isitman AT. Diagnostic bone scanning in oncology. Semin Nucl Med. 1997;27(2):107-41.

2. Hamaoka T, Madewell JE, Podoloff DA, Hortobagyi GN, Ueno NT. Bone imaging in metastatic breast cancer. J Clin Oncol. 2004;22:2942-53.

3. Shen G, Deng H, Hu S, et al. Comparison of choline-PET/CT, MRI, SPECT, and bone scintigraphy in the diagnosis of bone metastases in patients with prostate cancer: a meta-analysis. Skeletal Radiol. 2014:43(11):1503-13.

4. Römer W, Nömayr A, Uder M, Bautz W, Kuwert T. SPECT-guided CT for evaluating foci of increased bone metabolism classified as indeterminate on SPECT in cancer patients. J Nucl Med. 2006;47(7):1102-6.

5. Utsunomiya D, Shiraishi S, Imuta M, Tomiguchi S, Kawanaka K, Morishita S, et al. Added value of SPECT/CT fusion in assessing suspected bone metastasis: comparison with scintigraphy alone and nonfused scintigraphy and CT. Radiology. 2006;238:264-71.

6. Even-Sapir E. Imaging of malignant bone involvement by morphologic, scintigraphic, and hybrid modalities. J Nucl Med. 2005;46(8):1356-67.

7. Damle NA, Bal C, Bandopadhyaya GP, Kumar L, Kumar L, Kumar P, Malhotra A, et al. The role of 18F-fluoride PET-CT in the detection of bone metastases in patients with breast, lung and prostate carcinoma: a comparison with FDG PET/CT and 99mTc-MDP bone scan. Jpn J Radiol. 2013:31(4):262-9.

8. Cachovan M, Vija AH, Hornegger J, Kuwert T. Quantification of ${ }^{99 \mathrm{~m} T C-D P D}$ concentration in the lumbar spine with SPECT/CT. EJNMMI Res. 2013:3(1):45.

9. Beck M, Sanders JC, Ritt P, Reinfelder J, Kuwert T. Longitudinal analysis of bone metabolism using SPECT/CT and ${ }^{99 \mathrm{~m} T \mathrm{C}-}$

diphosphonopropanedicarboxylic acid: comparison of visual and quantitative analysis. EJNMMI Res. 2016;6(1):60.

10. Ritt P, Vija AH, Hornegger J, Kuwert T. Absolute quantification in SPECT. Eur J Nucl Med Mol Imaging. 2011;38(Suppl 1):S69-77.

11. Bailey DL, Willowson KP. An evidence-based review of quantitative SPECT imaging and potential clinical applications. J Nucl Med. 2013;54(1):83-9.

12. Seret A, Nguyen D, Bernard C. Quantitative capabilities of four state-of-theart SPECT-CT cameras. EJNMMI Res. 2012;2(1):45.

13. Kuwert T. Skeletal SPECT/CT: a review. Clin Transl Imaging. 2014;2:505-17.

14. Ross JC, Vilić D, Sanderson T, Vöö S, Dickson J. Does quantification have a role to play in the future of bone SPECT? Eur J Hybrid Imaging. 2019;3:8.

15. Dickson J, Ross J, Vöö S. Quantitative SPECT: the time is now. EJNMMI Phys. 2019;6:4.

16. Israel O, Pellet O, Biassoni L, De Palma D, Estrada-Lobato E, Gnanasegaran G, et al. Two decades of SPECT/CT - the coming of age of a technology: an updated review of literature evidence. Eur J Nucl Med Mol Imaging. 2019; 46(10):1990-2012.

17. Mariani G, Strauss HW. Positron emission and single-photon emission imaging: synergy rather than competition. Eur J Nucl Med Mol Imaging. 2011:38(7):1189-90.

18. van der Vos CS, Koopman D, Rijnsdorp S, Arends AJ, Boellaard R, van Dalen $J A$, et al. Quantification, improvement, and harmonization of small lesion detection with state-of-the-art PET. Eur J Nucl Med Mol Imaging. 2017; 44(Suppl 1):4-16.

19. Tsui BMW, Zhao X, Frey EC, Gullberg GT. Comparison between ML-EM and WLS-CG algorithms for SPECT image reconstruction. IEEE Trans Nucl Sci. 1991;38:1766-72.

20. Onoguchi M, Konishi T, Shibutani T, Matsuo S, Nakajima K. Technical aspects: image reconstruction. Ann Nucl Cardiol. 2016;2:68-72.

21. Vija AH. Introduction to XSPECT technology: evolving multi-modal SPECT to become context-based and quantitative. In: Vija AH (ed) Molecular Imaging White Paper: Siemens Medical Solutions USA, Inc., Molecular Imaging 2014.

22. Ma J, Vija AH. Evaluation of quantitation accuracy for xSPECT. Paper presented at: Nuclear Science Symposium and Medical Imaging Conference (NSS/MIC), 2015 IEEE, 2015. doi: https://doi.org/10.1109/NSSMIC.2015. 7582030.

23. Duncan I, Ingold N. The clinical value of XSPECT/CT Bone versus SPECT/CT. A prospective comparison of 200 scans. Eur J Hybrid Imaging. 2018;2(1):4.

24. Delcroix O, Robin P, Gouillou M, Le Duc-Pennec A, Alavi Z, Le Roux PY, et al. A new SPECT/CT reconstruction algorithm: reliability and accuracy in clinical routine for non-oncologic bone diseases. EJNMMI Res. 2018;8(1):14.

25. Kuji I, Yamane T, Seto A, Yasumizu Y, Shirotake S, Oyama M. Skeletal standardized uptake values obtained by quantitative SPECT/CT as an 
osteoblastic biomarker for the discrimination of active bone metastasis in prostate cancer. Eur J Hybrid Imaging. 2017;1 (1):2.

26. Willowson K, Bailey DL, Baldock C. Quantitative SPECT reconstruction using CT-derived corrections. Phys Med Biol. 2008;53(12):3099-112.

27. Tran-Gia LM. Characterization of noise and resolution for quantitative ${ }^{177} \mathrm{Lu}$ SPECT/CT with xSPECT quant. J Nucl Med. 2019;60:50-9.

28. Armstrong IS, Hoffmann SA. Activity concentration measurements using a conjugate gradient (Siemens xSPECT) reconstruction algorithm in SPECT/CT. Nucl Med Commun. 2016;37(11):1212-7.

29. Vija AH. Characteristics of the XSPECT reconstruction method. Siemens molecular imaging white paper. 2017.

30. Nakahara T, Daisaki H, Yamamoto Y, limori T, Miyagawa K, Okamoto T, et al. Use of a digital phantom developed by QIBA for harmonizing SUVS obtained from the state-of-the-art SPECT/CT systems: a multicenter study. EJNMMI Res. 2017;7(1):53

31. Zimmerman BE, Cessna JT. Development of a traceable calibration methodology for solid ${ }^{68} \mathrm{Ge} /{ }^{68} \mathrm{Ga}$ sources used as a calibration surrogate for ${ }^{18} \mathrm{~F}$ in radionuclide activity calibrators. J Nucl Med. 2010;51(3):448-53.

32. Miyaji N, Miwa K, Wagatsuma K, Umeda T, Murata T, Takiguchi T, et al. Quality control of dose calibrator using a traceable syringe-type ${ }^{68} \mathrm{Ge} /{ }^{68} \mathrm{Ga}$ calibration source. Nihon Hoshasen Gijutsu Gakkai Zasshi. 2013;69(12):137986.

33. Miyaji N, Miwa K, Motegi K, Umeda T, Wagatsuma K, Fukai S, et al. Validation of cross-calibration schemes for quantitative bone SPECT/CT using different sources under various geometric conditions. Nihon Hoshasen Gijutsu Gakkai Zasshi. 2017;73(6):443-50.

34. Ichikawa H, Miwa K, Matsutomo N, Watanabe Y, Kato T, Shimada H. Development of a novel body phantom with bone equivalent density for evaluation of bone SPECT. Nihon Hoshasen Gijutsu Gakkai Zasshi. 2015; 71(12):1235-40.

35. Iida H, Hori Y, Ishida K, Imabayashi E, Matsuda H, Takahashi M, et al. Threedimensional brain phantom containing bone and grey matter structures with a realistic head contour. Ann Nucl Med. 2013:27(1):25-36.

36. Kaneta T, Ogawa M, Daisaki H, Nawata S, Yoshida K, Inoue T. SUV measurement of normal vertebrae using SPECT/CT with Tc-99 m methylene diphosphonate. Am J Nucl Med Mol Imaging. 2016;6(5):262-8.

37. Akamatsu G, Ikari Y, Nishida H, Nishio T, Ohnishi A, Maebatake A, et al. Influence of statistical fluctuation on reproducibility and accuracy of SUVmax and SUVpeak: a phantom study. J Nucl Med Technol. 2015;43(3): 222-6.

38. Soret M, Bacharach SL, Buvat I. Partial-volume effect in PET tumor imaging. J Nucl Med. 2007:48(6):932-45.

39. Shinohara $H$, Hashimoto $T$. An error evaluation of iterative image reconstruction methods using chi-square $(\mathrm{x} 2)$ statistic minimization for Poisson-distributed projection data. Igaku Butsuri. 2018;38(3):113-28.

40. Okuda K, Fujii S, Sakimoto S. Impact of novel incorporation of CT-based segment mapping into a conjugated gradient algorithm on bone SPECT imaging: fundamental characteristics of a context-specific reconstruction method. Asia Ocean J Nucl Med Biol. 2019;7(1):49-57.

41. Matsutomo N, Matsumoto S, Yamamoto T, Sato E. Validation of a calibration method using the cross-calibration factor and system planar sensitivity in quantitative single-photon emission computed tomography imaging. Radiol Phys Technol. 2017;10(4):439-45.

\section{Publisher's Note}

Springer Nature remains neutral with regard to jurisdictional claims in published maps and institutional affiliations.

\section{Submit your manuscript to a SpringerOpen ${ }^{\circ}$ journal and benefit from:}

- Convenient online submission

- Rigorous peer review

- Open access: articles freely available online

- High visibility within the field

- Retaining the copyright to your article

Submit your next manuscript at $\boldsymbol{\nabla}$ springeropen.com 